\title{
Research on the Teaching Model of Animation Professional Class Based on AR/VR Technology and 5G Network
}

\author{
Lixin Fu \\ School of Design Art and Architecture, Zhejiang Wanli University, Ningbo 315100, China \\ Correspondence should be addressed to Lixin Fu; fulixin2021@126.com
}

Received 27 October 2021; Revised 29 November 2021; Accepted 30 November 2021; Published 26 December 2021

Academic Editor: Xin Ning

Copyright (c) 2021 Lixin Fu. This is an open access article distributed under the Creative Commons Attribution License, which permits unrestricted use, distribution, and reproduction in any medium, provided the original work is properly cited.

\begin{abstract}
The use of emerging technologies is becoming more common in today's world, and as media integration deepens, so does the use of virtual reality technology. As an emerging science and technology, VR/AR technology plays a critical role in society's development and progress, and its application in current education and teaching has become an indispensable link. The production of high-quality animation professionals is the society's and the animation industry's urgent need for talent, the precondition for the prosperity of cultural and creative industries, and the foundation of national soft power. To bring more technical changes to education and provide theoretical support for the realization of human-computer interaction technology, this paper mainly elaborates on the concept and application fields of VR/AR technology and discusses the specific applications of VR/AR technology according to its different educational stages. The main point of the presentation is to demonstrate how, in the era of $5 \mathrm{G}$ networks, large bandwidth, ultralow latency, massive connectivity, and ultrahigh reliability provide a strong network foundation for widespread VR/AR application. We will investigate the paucity of teaching methods, teaching modes, and teaching platforms as a result of the application of 5G-based VR/AR technology in the teaching field of animation majors, as well as the design of teaching methods, teaching modes, and experimental training platforms based on 5G-based VR/AR technology in animation majors in conjunction with the training plan of engineering students at our university. We will delve deeper into the integration of VR/AR technology and higher education teaching, laying the groundwork for "AI+" education. VR/AR technology can be used in the classroom to present information to students in a more three-dimensional and intuitive way, which not only increases student interest in learning but also improves the learning experience and cultivates core literacy.
\end{abstract}

\section{Introduction}

The concept of virtual reality (or VR for short) became widely known in 2016, the "Year of VR." The concept is not a new one in recent years [1], as its products date back to 1968 with Ivan Sutherland's "Sword of Damocles," which is considered to be the world's first VR device. The term "virtual reality" was first coined by the founder of VPL in the early 1980s, and the concept was defined in a way that pushed the research and development of VR technology into a new phase. Commercialization of VR has been attempted for more than 20 years, starting with Sega's 1991 VR arcade game, but most attempts have failed due to the immaturity of the technology. In recent years, with technological advances and capital pushing VR devices toward refinement [2], lightness, and mass appeal, the scale of commercializa- tion attempts on VR has gradually expanded, starting with attempts in gaming and beginning with various convergence attempts in healthcare, real estate, traditional retail, education, film, and travel. Facebook acquired VR wearable device maker Oculus for $\$ 2$ billion in 2014, a move that intensified capital attention and investment in the VR industry, which finally entered a complete explosion in 2016 after reaching a total market value of $\$ 1.8$ billion [3].

Nowadays, there is a demand for accelerating the construction of intelligent learning systems in universities for next-generation networks, adapting to the development of $5 \mathrm{G}$ network technology, serving the new requirements of intelligent learning in all-time, all-space, and all-audience, promoting the revolution of classroom teaching, vigorously promoting the construction of intelligent classrooms, building a teaching model combining online and offline, actively 
guiding students to self-management and active learning, stimulating the desire for knowledge, improving learning efficiency, and enhancing independent learning ability. In addition, I will enhance my ability of independent learning. Using 3D modeling, 3D virtual development engine, database, network communication, and other related knowledge, I reveal the feasibility of using $5 \mathrm{G} \mathrm{VR} / \mathrm{AR}$ technology to build a virtual equipment platform for experimental training and promote students' personalized development of learning methods. Then, based on the theoretical basis that virtual classrooms and real classrooms cooperate, I adopt 5G technology to solve the problem of learning anywhere and anytime and build a classroom teaching design method suitable for this technology. Finally, I will synthesize and summarize traditional teaching methods and teaching models and build a higher education teaching scenario with the characteristics of 5G VR/AR technology [4]. The theoretical framework, teaching design, and practical training platform for the application of $5 \mathrm{G}$ VR/AR technology in higher education will be discussed from the perspective of the generality and practicality of the application of $5 \mathrm{G} \mathrm{VR}$ / AR technology extracted from the classroom.

In the past, the teaching mode of animation professional class corresponds to the traditional education teaching in schools, but starting from online teaching using information technology, traditional education gradually integrates and develops with emerging technology, which in turn changes traditional teaching, and the teaching mode of animation professional class is transformed and upgraded, such as developing digital teaching materials. Under the influence of VR/AR technology, there will be a new transformation of traditional education and teaching. At present, VR/AR is still limited by technical problems such as hardware. When the hardware technology is effectively solved, VR/AR technology will be more popular and will provide a new round of transformation power for the traditional education and teaching mode, and the change will then affect the transformation of the animation professional class teaching mode field. At the same time, VR/AR education needs to have supporting digital animation professional class teaching mode to provide content support, and in the face of the wave of emerging technology, the content of traditional education will also seek recreation through VR/AR, which will also bring new market space for the field of animation professional class teaching mode. In recent years, the situation of traditional paper media is still difficult. In essence, VR/AR is just a carrier, and without content, it cannot directly address the audience [5]. Publishing companies, on the other hand, have professional content and have the advantage of integrating with VR/AR. Some publishing companies have already started to explore the integration of content and VR/AR and further explore new models of profitability and services. Many publishers, especially local publishers, rely on the animation professional teaching model and see the digital animation professional teaching model as an important time for transformation. The wave of VR/AR emerging technologies has prompted the animation professional teaching model to move further into the VR/AR animation professional teaching model. This transformation will not only provide innovative content carriers for traditional textbooks and teaching aids but also expand the boundaries of animation professional teaching models.

\section{Related Work}

Facing the challenges and opportunities brought by such emerging technologies, VR/AR integration attempts, among which building a VR/AR digital educational publishing platform is an option for many educational publishing organizations [6]. The article "The Application and Development Strategy of VR/AR Technology in Digital Educational Publishing Platform" argues that the immersive, contextual, and interactive characteristics of VR/AR technology contribute to the scenario-based, three-dimensional, and interactive characteristics of digital educational publishing platforms and have inherent advantages when applied to digital educational publishing platforms. In terms of VR/AR digital educational publishing platform development methods, there are usually two kinds, namely, VR/AR digital educational publishing platform development mainly has two kinds of independent construction and cooperative construction [7]. In September 2011, a developed country released its "Smart Education Strategy," which plans to build a new education ecosystem from three aspects: "upgrading infrastructure, reforming the education system, and enhancing teachers' capacity," using Internet+Cloud Classroom, Internet+Electronic Textbook, and Internet+Online Assessment to build a new education ecosystem. In 2012, country A released its "Transformative Education," which proposes to use information technology to improve the capacity of teachers and education administrators, introduce interactive classrooms and other online learning methods into traditional teaching and learning, and develop a new-age workforce with global skills [8].

The Federal Ministry of Education of State B has proposed a K-12 education reform strategy centered on smart education; State A has proposed a smart education solution centered on "building an online data system, sharing a library of teaching resources, and building a social network for teachers." According to the literature, AI should be widely used in education and that the time is now. The combination of education and robotics will present a new way of teaching and learning in the classroom of the future in the next 25 years [9], assisting teachers in improving their teaching and assisting students in participating well in classroom learning, as well as improving classroom efficiency. The literature claims that Internet information technology has influenced a variety of areas in today's world, and the researcher's goal of combining Internet technology with new teaching methods in the understanding and development of the concept of smart teaching and learning is supported by the literature. According to the literature, new teacher induction learning, teaching methods, and approaches, as well as the use of smart tools, will play a critical role in the transition from traditional to smart education. From a practical standpoint, some international technology companies are committed to designing smart education solutions and building smart education teaching 
models. For example, IBM's five smart education solutions and Samsung's Smart Education Cloud Platform in South Korea have been implemented at North Carolina State University as well as primary and secondary schools in the country. Kumara and W.W. et al. argue that as humancomputer interaction improves, humans will be able to interact with microcomputers or smartphones through body movements and that the combination of body perception and in-class learning will improve learners' learning experience and process [10].

The researcher designed an evaluation system in smart teaching and learning by making an experimental sample of teachers and students and analyzing the feedback results of the sample in conjunction with a validity questionnaire. The results showed that the participants were satisfied with the simple and easy-to-operate system, and the evaluation results showed that this system stimulates students' curiosity to learn [11]. The literature reflects the design of a deep simulation and inquiry organization for smart technology classrooms in its study. Using a collaborative group design approach, a virtual simulation of a bank was created in collaboration with a vocational school teacher organization, and a group of giant screen monitors was set up in the lab to engage and infiltrate the students. The results of the experiment showed that the majority of students who participated and infiltrated the experiment were able to construct models and articulate their ideas about the banking environment [12]. The literature provides a clear analysis of the effectiveness of smart teaching and learning to investigate the perceptions of vocational school students about smart teaching and learning. The researcher selected a random sample of 100 vocational school students from two Indian vocational schools for the study and analyzed the perception results of these respondents through a chi-square experiment and univariate factorial technique [13]. The results of the study showed that traditional teaching methods, teachers' knowledge base, demonstrated skill approach, usage of classroom blackboard, elaboration of cases, classroom questions, review and consolidation of knowledge, and feedback on assignments are much better when combined with smart teaching methods [14]. The advent of the "Internet+" era, the impact of big data, cloud platforms, and other wisdom teaching backgrounds on education has attracted the attention of domestic experts and scholars, who have launched an in-depth exploration of wisdom teaching in theory and practice under the background of "Internet+." They have conducted in-depth theoretical and practical research on intelligent teaching in the context of "Internet+" [15].

\section{Research on Teaching Mode of Animation Professional Class Based on AR/VR Technology and 5G Network}

3.1. AR/VR and the Application of 5G Network Technology. Virtual reality (VR) is an application that generates a three-dimensional virtual space for users through computer technology, including visual, tactile, auditory, and other sensory simulations, and users can enter and interact with the virtual space with the help of specific input and output devices. The "reality" in "virtual reality" refers to any environment or thing in the world, which can be a reality that exists or can be achieved, or a reality that is difficult or impossible to achieve; and "virtual" stands for computergenerated. In essence, virtual reality is an advanced computer user interface that provides users with a means of instant interaction with natural images across multiple senses [16]. VR has the " $3 I$ " characteristics of immersion, conceptualization, and interactivity. Currently, there are three mainstream types of VR devices around the world: VR headsets, which must be connected to a PC when in use, such as Oculus Rift, HTC Vive, 3Glasses, and other typical products, and gaming headset VR devices, which can be connected to a game console, such as Sony PS VR and Microsoft VR platform; this type of VR headset is not easy to move, has space constraints, and is expensive. But it is currently the best immersive experience among the three types of devices. The second is the VR all-in-one; this type of device built-in processor, display and other hardware, the use of lighter and more flexible, can be carried around, and can bring users a better VR immersion experience, but the development and production of VR all-in-one technology are not yet mature and expensive, small school VR, Qiwei VR, DaBeng VR, and other technology companies, is the development of such devices on behalf of the company. Google's Cardboard is the earliest carton product, while mobile VR is the development of improved VR cartons, the VR carton with a design shell and more professional optics so that it has a stronger sense of design and functionality than the carton, typical products such as NOLO VR. The mathematical formula is as follows.

$$
E(t)=A(t) X(t)-\frac{1}{2} \frac{n !}{r !(n-r) !}
$$

The first formula in the article is closely related to the article. Based on the algorithm used in this article, it improves animation teaching and greatly improves efficiency. The disadvantage of this type of VR device is that its immersion effect is not ideal, but the advantage is the low price, flexibility, convenience, ease to carry, etc. and is currently the best-selling VR device in the market; it lowers the threshold for the public to VR experience, but with the progress of technology, VR market maturity, the sales of such entry devices will gradually decline. Augmented reality (AR) is a modern technology that generates virtual objects that do not exist in the physical world with the help of computer graphics and visualization technology and places them exactly in the physical world, superimposing virtual information to the real world in real time so that both exist simultaneously in the same plane or space, which can provide users with a more perceptually richer reality. AR technology uses location tracking devices to obtain interaction data used by the user to achieve the user's command to manipulate virtual objects. AR technology can achieve the effect of combining reality and human-computer interaction and 


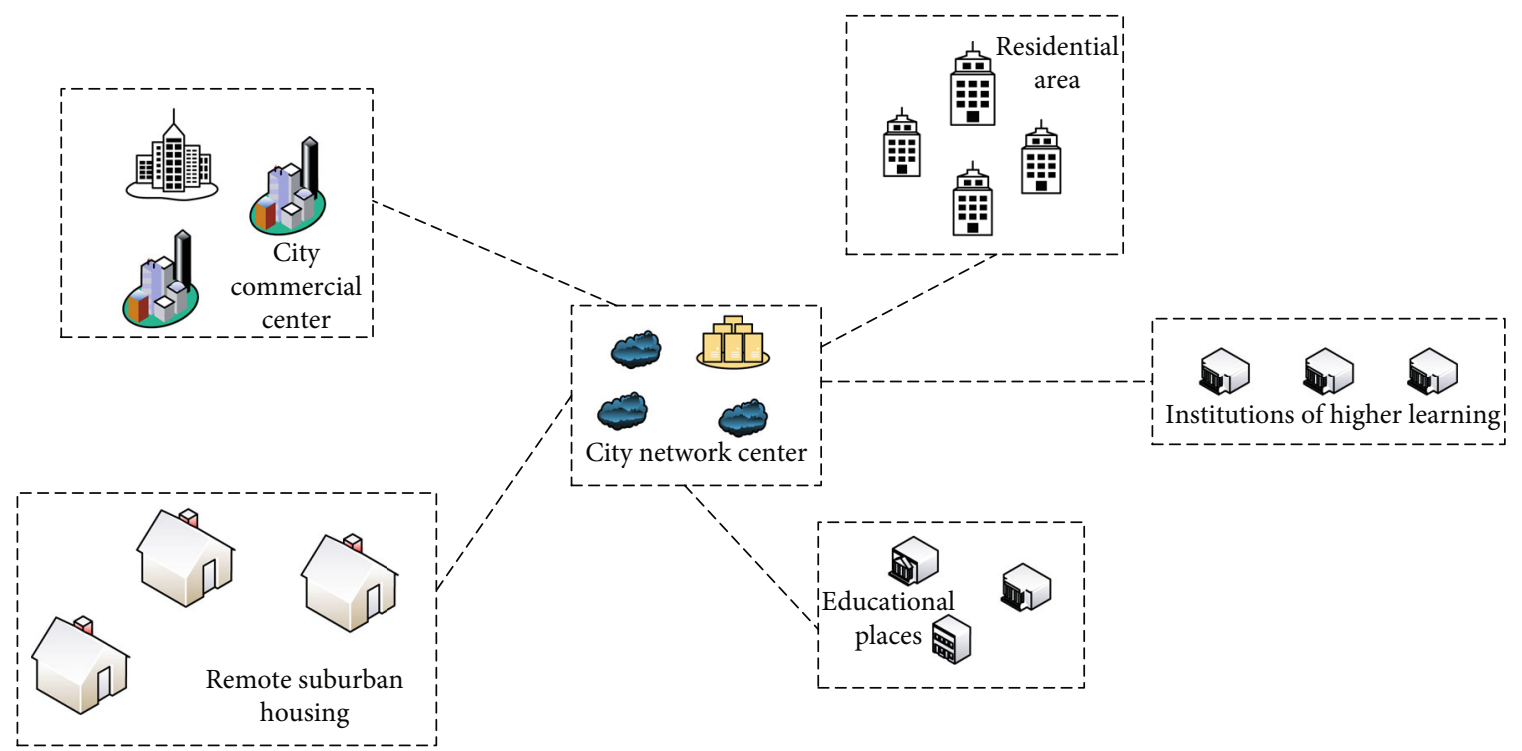

FIGURE 1: Future 5G network usage scenarios.

has three characteristics of combining reality and reality, three-dimensional registration, and real-time interaction or instant interaction. AR functions are mainly realized through AR glasses, such as Google Glass, and can also be realized by using mobile terminal devices, such as tablets and smartphones. The principle of the mathematical formula is as follows [17].

$$
E(r)=\frac{\partial^{2} \Omega}{\partial u \partial v}-\arcsin \theta
$$

5G technology can effectively improve the spectral efficiency and link reliability of the system by employing multiple antenna arrays at the transceiver end and using the variability of channel fading at different antenna array locations to obtain spatial diversity or multiplexing gain, which has been widely used in communication systems in recent years [18]. The key technology in 5G and B5G systems, compared to the traditional 2D5G technology, extends the arrangement of antenna arrays to $3 \mathrm{D}$ space and flexibly controls the weights of each array element in $3 \mathrm{D}$ space to achieve $3 \mathrm{D}$ beam assignment, thus further enhancing the spectral efficiency of the system. 5G later use scenarios are shown in Figure 1.

The performance of $5 \mathrm{G}$ technology mainly depends on the correlation between channel characteristics and antennas; therefore, for 3D 5G technology, an in-depth study of 3D 5G channels is needed, especially to consider the new characteristics of the added vertical dimension of the channel and its impact on the performance of the communication system. New features of the application of 3D 5G technology may be reflected in the following aspects: (1) diverse typical new scenarios: future 5G and B5G. Typical scenarios for mobile communication system applications include urban macrocells, urban microcells, and outdoor stations covering indoor and indoor hotspot scenarios. To improve the accuracy of channel modeling and to be able to evaluate the performance of transmission technologies in different scenarios, it is necessary to fully investigate and distinguish the differences in channel propagation characteristics in different scenarios. In particular, in 3D channels [19], the added vertical dimension makes the channel propagation characteristics in different scenarios very different. In a typical outdoor station covering an indoor scenario, users located on different floors can be distinguished by the height difference of the vertical dimension, and then, the $3 \mathrm{D}$ beam assignment can be used to achieve dynamic coverage for users, thus improving the system capacity. In the indoor scenario, the placement pattern of the $3 \mathrm{D}$ antenna array also has an impact on the propagation characteristics of the multiplier channel. In the out-of-beam scenario, higher multiplexing gain can be provided to small K-edge users by using 3D 5G vertical dimensional beam assignment. The principle of the formulation is as follows.

$$
M(t)=\left(\begin{array}{cc}
1 & 0 \\
2 & -1
\end{array}\right) .
$$

(2) Multiuser communication: using the increased vertical dimension in the transceiver: three-dimensional space, 3D 5G technology allows more antenna arrays to be arranged in a limited space. Vertical dimensional beam assignment is also used to better distinguish different users, allowing 3D 5G technology to achieve simultaneous communication of multiple users, effectively increasing system throughput. Furthermore, 3D 5G technology allows for more antenna arrays to be arranged at the base station, and the system is more stable when the number of antenna arrays is large. When the number of antenna arrays at the base station exceeds the number of users [20], the system has a large number of degrees of freedom, allowing interference between users to be eliminated using the interference cancellation algorithm. Furthermore, increasing the number of antenna arrays can result in higher diversity gain, reducing the impact of Gaussian white noise on system 


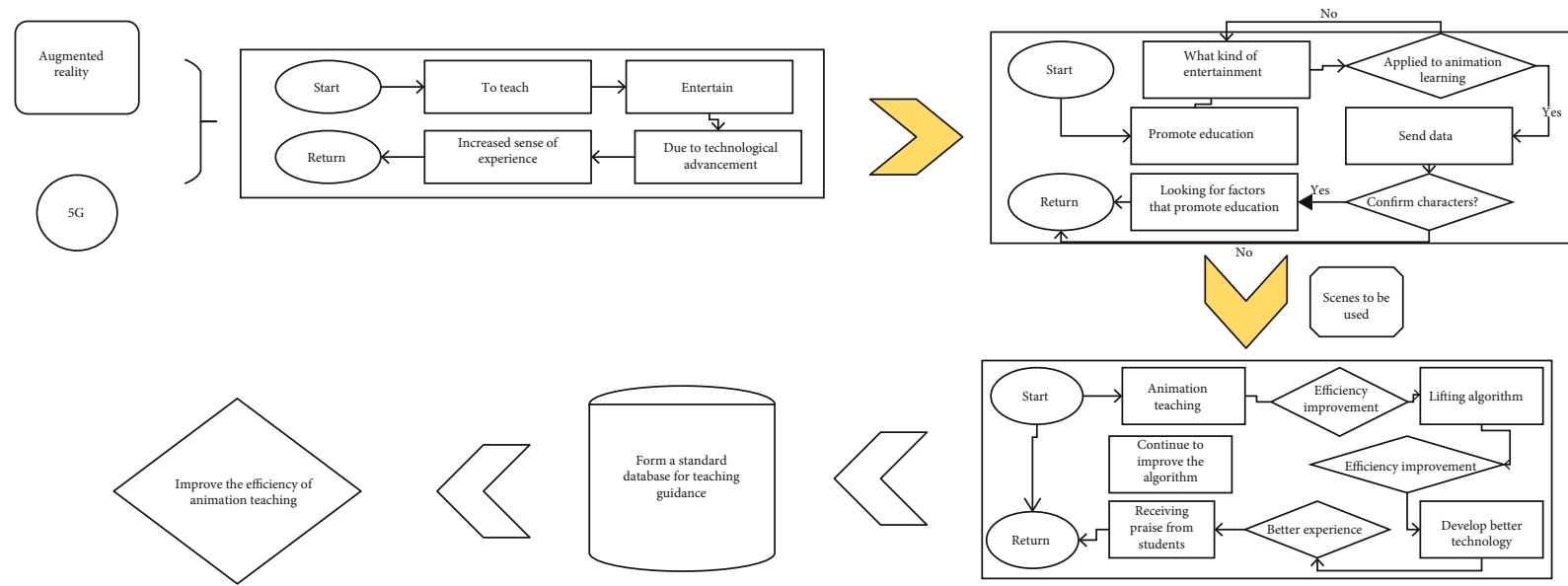

FIGURE 2: Multiuser communication mode.

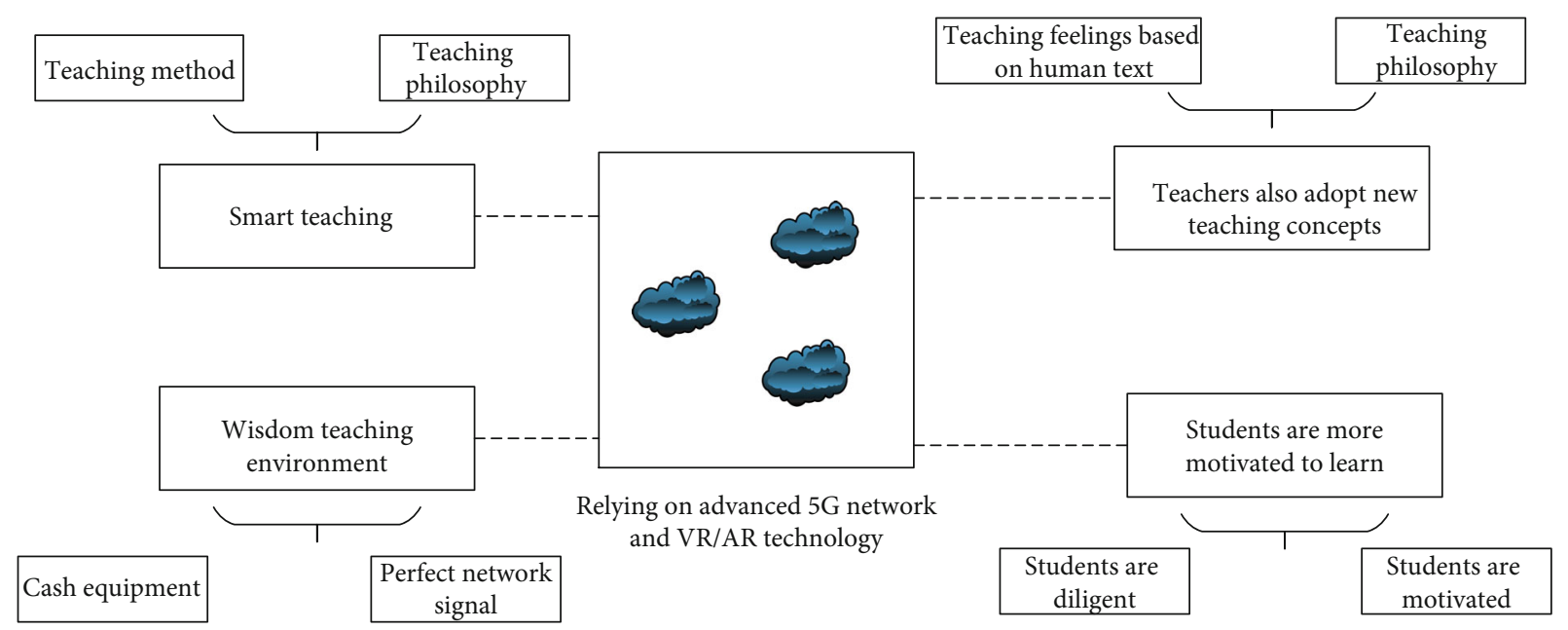

Figure 3: Framework for smart education research.

performance and improving system stability. Figure 2 illustrates an example of multiuser communication.

(3) Millimeter-wave and terahertz higher frequency bands: communication technologies in the higher frequency bands of millimeter-wave and terahertz can provide greater bandwidth compared to sub- $6 \mathrm{GHz}$, which in turn enhances the system capacity PW. In addition, the shorter wavelengths of millimeter-wave and terahertz higher frequency bands can be used to arrange more array elements in a limited antenna array space, which, combined with the spatial expansion of the vertical dimension of 3D technology, opens up the possibility of forming $3 \mathrm{D}$ superscale $5 \mathrm{G}$ array become possible. With 3D large-scale 5G technology, interference between users can be eliminated by simple precoding of different users, enabling simultaneous multiuser communication and significantly increasing system capacity.

In 5G and B5G systems, 3D 5G as a key technology will further enhance the spectral efficiency and throughput of the system by combining with technologies such as millimeterwave and terahertz higher frequency bands and massive 5G. In particular, in more application scenarios, 3D 5G technology can provide additional multiplexing gain using the increased vertical dimension, which in turn can support more users to communicate simultaneously, which is the current research focus of 3D 5G technology.

3.2. Study on the Teaching Mode of Animation Professional Class. The framework for research on smart education consists of five main components: smart educational philosophy, smart environment, smart pedagogy, smart assessment, and smart talent (called smart learners in school contexts). They correspond to educational philosophy innovation, technological innovation, methodological innovation, assessment innovation, and talent perspective change, respectively. The relationship between them is that the concept of smart education plays an overarching and leading role in the research framework, which directly points to a fundamental change in the view of talent in the digital age and enables the cultivation of smart learners through the "hard" smart learning environment and "soft" strategies and methods. The logical relationship of this framework can be further expressed as follows: using appropriate information technology to build a smart learning environment (technological innovation), applying smart pedagogy 

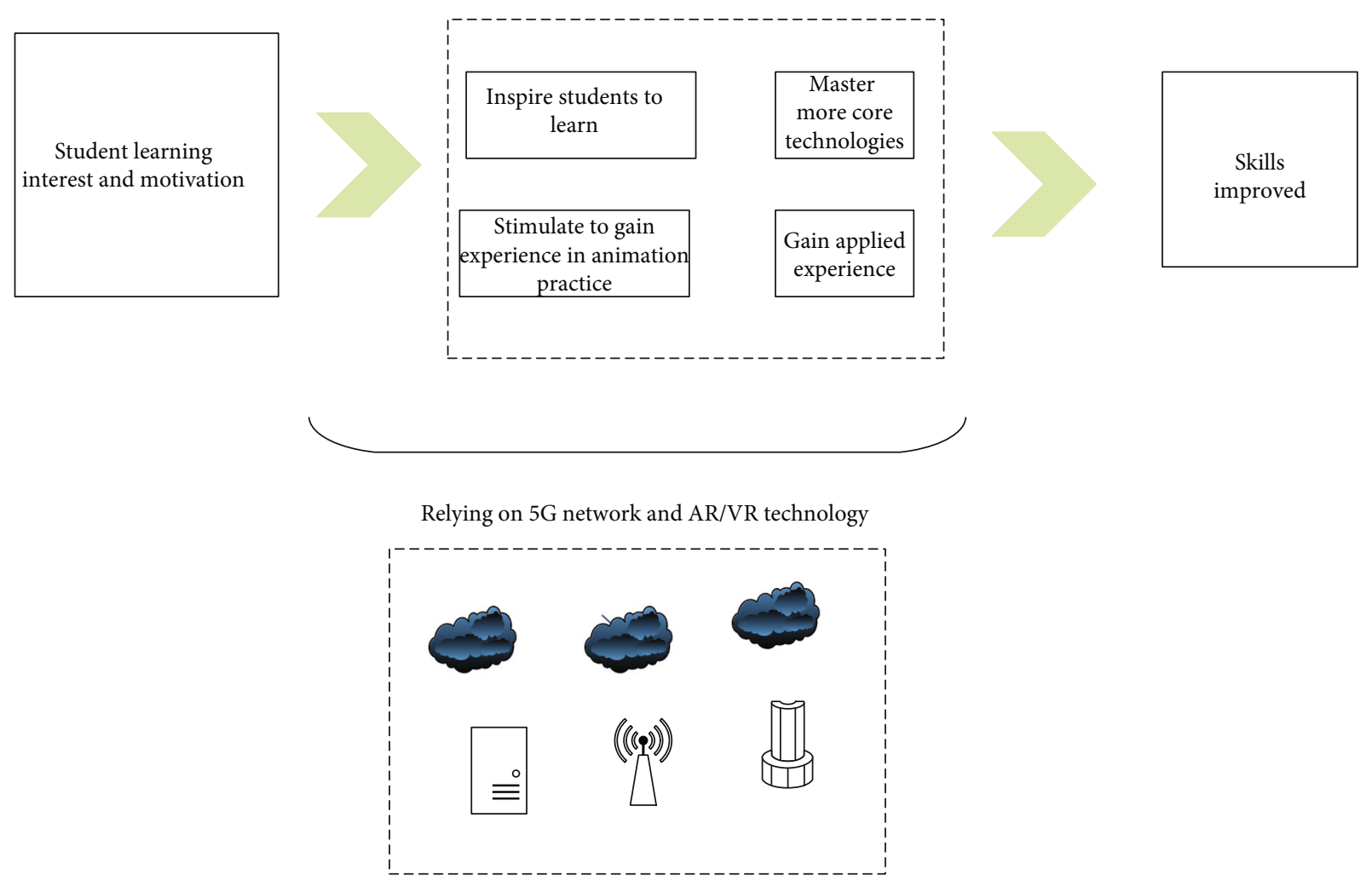

FIGURE 4: The integrated practice teaching model.

(pedagogical innovation), facilitating smart learning by learners (pedagogical innovation), enabling learners to receive appropriate personalized learning services and wonderful developmental experiences (service innovation), and implementing accurate and panoramic smart evaluation (evaluation innovation); to cultivate future-oriented wise learners who can cope with complex challenges, solve complex problems, and have virtues and good deeds (talent concept change), continuously implement the concept of wisdom education (concept innovation) and deepen and enhance quality education in the information age, knowledge age, and digital age. Its framework diagram for wisdom education research is shown in Figure 3.

To guide the assessment of the effectiveness of the development of wise learners (expert learners) and facilitate the development of their wisdom abilities in a targeted and focused manner in teaching practice, this study constructs a model of wise learners' abilities in Chapter 1. The model consists of five levels: (1) personal level: mainly including good use of technology, good learning, and good selfmanagement; (2) social level: mainly including good communication and good collaboration; (3) application level: mainly including good practice and good problem solving; (4) scientific innovation level: mainly including good research and judgment and good innovation; (5) value experience level: having correct values, positive learning attitudes, and a strong sense of social responsibility.

The animation industry is a collection of industries related to animation, including the development, production, publication, broadcasting, performance, and sales of various products such as books, films, stage productions, and information dissemination equipment. It is based on character images as a brand endorsement, animation broadcast or publication as a form of expression, and creativity and realization of content as product value. The industry is generally divided into two development models. One is the technology economy model, which is mainly to meet the needs of the product; and another is the market economy model, which is mainly centered on consumer demand. If the animation industry is based on meeting the needs of products, then the amount of animation completed is an important indicator of the development of the animation industry, and the training of production personnel is the main training goal in animation education. However, the animation industry is a cultural and creative industry, which has to face consumers, guide them, and satisfy as well as expand their consumer psychology and needs. Therefore, animation education cultivates animation talents who are not only engaged in animation production but also meet the animation talents of each link in the industry chain. Its way of training students is integrated practical teaching, and its model diagram is shown in Figure 4.

\section{Experimental Results and Analysis}

4.1. Experimental Results. The summary statistics of each teacher's understanding of the connotations of the $5 \mathrm{E}$ teaching model in the interview transcripts revealed that all 10 teachers had some knowledge of the 5E teaching model, but the conceptual understanding was vague. Among them, six teachers had been exposed to the 5E teaching model through literature search and biology teaching-related 


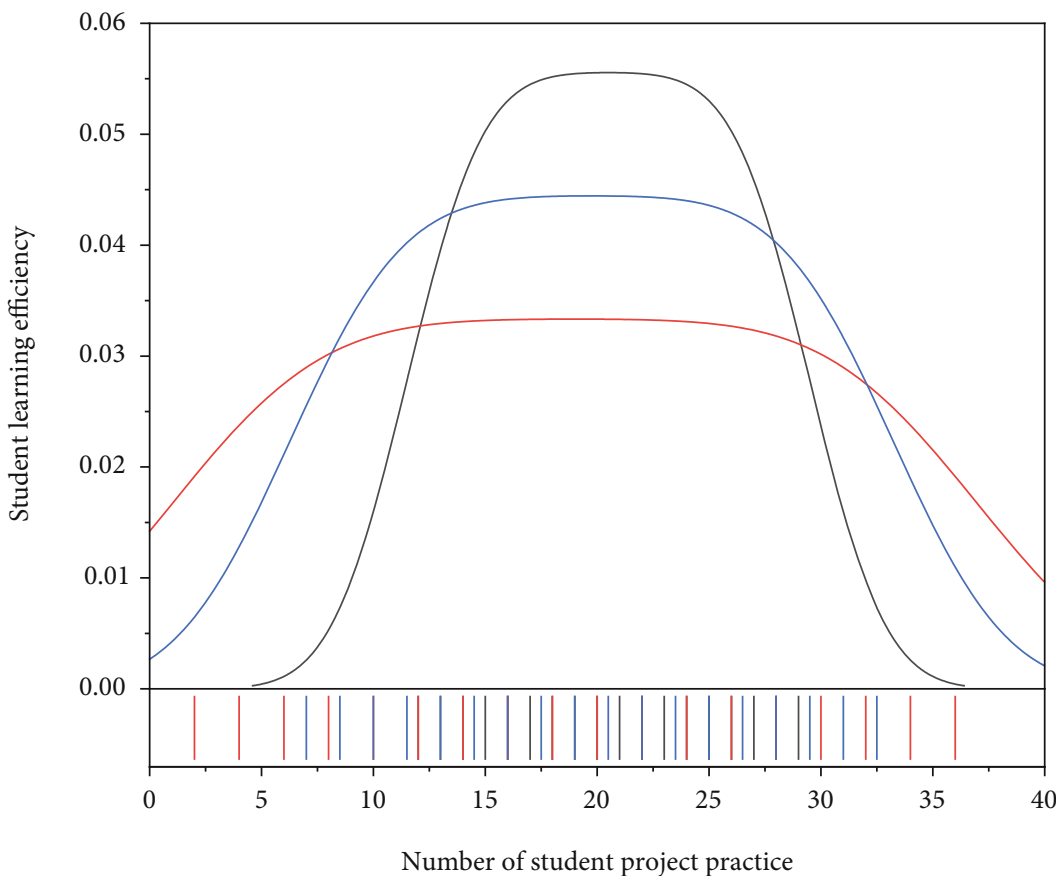

elying on $5 \mathrm{~g}$ network

- Relying on VR technology

_ Relying on AR technology

FIGURE 5: Comparison graph of experimental results.

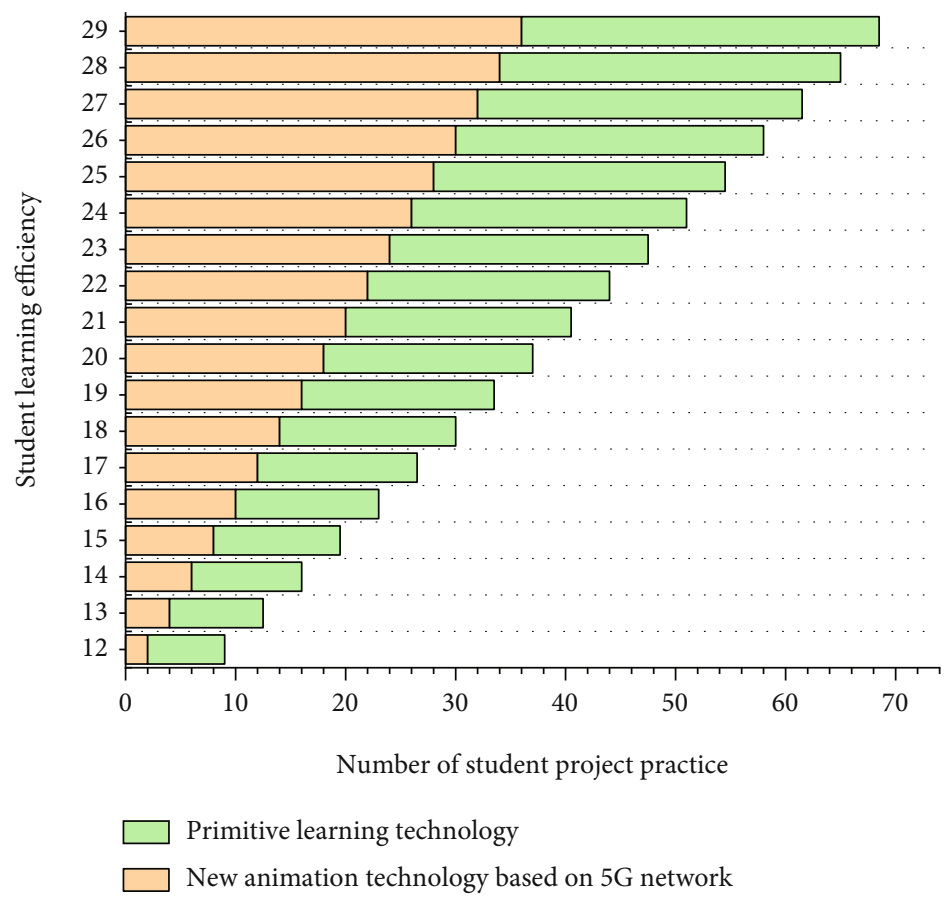

FIGURE 6: Graph showing the degree of improvement in inquiry capacity.

journals before conducting teaching and research activities and group lesson preparation. However, when asked about the elaboration of the aspects of the $5 \mathrm{E}$ teaching model, only one teacher could accurately describe the five aspects, and most teachers confused the conventional teaching aspects with the aspects of the $5 \mathrm{E}$ teaching model, such as attraction and introduction, explanation and teaching, and transfer and application. The summary statistics of each teacher's knowledge of the new teaching materials in the interview transcripts showed that 10 teachers expressed their own 


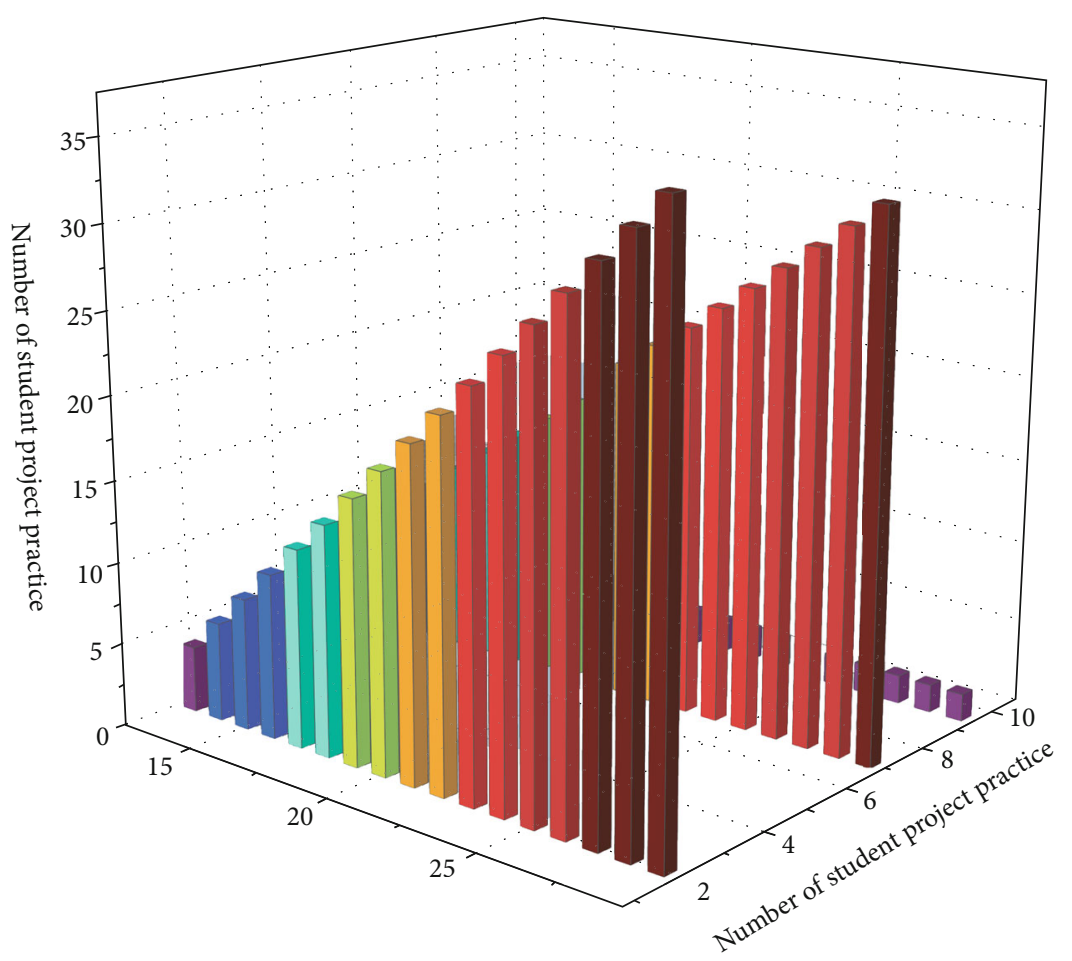

FIGURE 7: Graph of teaching efficiency improvement performance in animation professional category.

opinions and the classification was confusing. Most teachers classified knowledge from subjective assumptions and lacked systematic theoretical support, juxtaposing concepts, principles, experiments, calculations, applications, etc. Two teachers even confused knowledge, competence, and skills, and only one teacher classified knowledge into declarative knowledge, procedural knowledge, etc. based on relevant theories of educational psychology. A comparison of their experimental results is shown in Figure 5.

The implementation of the $5 \mathrm{E}$ teaching model with improved countermeasures for classroom teaching resulted in positive changes in many ways. On the one hand, the classroom atmosphere in the experimental classes improved significantly, students' motivation and initiative in answering questions increased, and the teaching sessions progressed more smoothly. On the other hand, students' abilities in many aspects were improved, especially their self-learning ability, cooperative learning ability, and hands-on ability. For example, when working in groups, each person has a clearer division of labor and can quickly grasp where the conflict lies, communicate and communicate intensely with the information at hand, and able to complete the task with high efficiency and quality within the time limit. In a comparison between the experimental class and the control class, the teacher interviews revealed that the students in the experimental class had improved in various aspects such as self-learning ability and cooperative learning ability; the paper-and-pencil test revealed that the students in the experimental class had made substantial progress in their academic performance and inquiry ability; the classroom observation revealed that the inquiry ability in the experimental class had improved significantly, and the degree of improvement is shown in Figure 6.

4.2. Experimental Analysis. This study draws on the psychological research results of "wisdom," especially the research results of learning science on expert learners, and lays the scientific foundation for the characterization of "wise (expert) learners." For the first time, a new taxonomy of educational goals and the definition of "acquired competencies" are used as methodological guidelines to describe acquired wisdom in terms of acquired competencies, and then, a model of wisdom (expert) learner competencies is proposed to enhance the relevance, effectiveness, and operability of wisdom education in the cultivation of wisdom (expert) learners. The study argues that wisdom education implies a profoundly new pedagogical structure-one that is guided and supported by the teacher and one that is conducive to knowledge innovation through students' autonomous study and active practice. On the one hand, the mainstream teaching model in schools has difficulty in realizing the pedagogical structure of smart education, which has significantly improved the efficiency of teaching and learning, as shown in Figure 7.

On the other hand, a brief review of differentiated instruction reveals that the differentiated instruction model has the possibility and feasibility of achieving a new pedagogical structure for smart education. To further enhance the power of the differentiated instructional model to achieve this new pedagogical structure, this study highlights the higher requirements of smart education for it, such as deep integration of technology, constructing expert 
knowledge, teaching-learning strategies, and data-based decision-making. For instructional model design, as a purposeful and creative activity, based on the aforementioned possibilities and feasibility analysis, this study redesigned technology-supported differentiated instructional models to achieve instructional structures that are aligned with smart education and transformed through instructional structures to improve intellectual competencies, enhance learning experiences, and improve academic performance. The research process was generally conducted in two rounds. In the first exploratory research process, the researcher mainly observed and analyzed the learning platform records, classroom behavioral performance, quality of academic work, and the effects of instructional interventions and further adjusted and optimized the differentiated instructional model based on the analysis results. In the second formal research phase, the effectiveness of the application of the improved differentiated teaching model in subject teaching was reviewed. To this end, three main hypotheses are proposed: (1) the differentiated teaching model is more advantageous than the traditional teaching model in improving students' academic performance; (2) the differentiated teaching model is more advantageous than the traditional teaching model in improving students' intellectual ability for the 21 st century and enhancing good learning experiences; (3) the differentiated teaching model is more advantageous than the traditional teaching model in changing the structure of classroom teaching.

\section{Conclusion}

Student data is an important foundation for differentiated instructional decisions in smart education. The nature (e.g., openness and complexity) and content (e.g., knowledge categories, knowledge capacity, and presentation) of learning tasks, the form of instructional organization (e.g., individual and small or large group), and the choice of instructional strategies, among other things, can be directly influenced by the results of analyzing relevant student data. Based on the results of student data analysis, this study constructively proposes a decision model for the application of differentiated instructional models, which can assist teachers in making appropriate arrangements for the nature of learning tasks, instructional organization forms, and instructional strategies in the process of applying differentiated instructional models. The differentiated teaching decision model is built around an accurate data-based decision-making mechanism. It is a crucial mechanism for aligning learning tasks, grouping formats, instructional strategies, and learning environment support based on the findings of multiple student data analyses in order to meet specific instructional goals and realize the structure of smart education instruction. To that end, the strategy matrix proposed in this study for the application of differentiated instructional models focuses on the nature of the task (simple, complex, and open) and the level of acquired competencies (high, medium, and low) and provides suggestions for specific instructional strategies and corresponding task arrangements to assist teachers in selecting the best combination of strategies. This paper uses new technologies that are more capable of enabling better teaching of animation classes, based on AR/VR technology and 5G networks. AR/VR technology and 5G technology should be mainly based on human-computer interaction experience in the future development direction so that people can get better development in technological innovation.

\section{Data Availability}

The data used to support the findings of this study are included within the article.

\section{Conflicts of Interest}

The author does not have any possible conflicts of interest.

\section{References}

[1] P. Madduru, "Artificial intelligence as a service in distributed multi access edge computing on $5 \mathrm{~g}$ extracting data using IOT and including AR/VR for real-time reporting," INFORMATION TECHNOLOGY IN INDUSTRY, vol. 9, no. 1, pp. 912931, 2021.

[2] Y. Zhang, Z. Feng, H. Moustafa, F. Ye, U. Javaid, and C. Cui, "Guest editorial: edge intelligence for beyond $5 \mathrm{G}$ networks," IEEE Wireless Communications, vol. 28, no. 2, pp. 10-11, 2021.

[3] J. S. Panchal, S. Subramanian, and R. Cavatur, "Enabling and scaling of URLLC verticals on 5G vRAN running on COTS hardware," IEEE Communications Magazine, vol. 59, no. 9, pp. 105-111, 2021.

[4] H. Zhou and L. Que, "The causes and enlightenment of postmodern film culture in the 5G era," Journal of Electronic Research and Application, vol. 5, no. 5, pp. 25-30, 2021.

[5] S. Mumtaz, V. G. Menon, and M. I. Ashraf, "Guest editorial: ultra-low-latency and reliable communications for $6 \mathrm{G}$ networks," IEEE Communications Standards Magazine, vol. 5, no. 2, pp. 10-11, 2021.

[6] S. Wijethilaka and M. Liyanage, "Survey on network slicing for Internet of Things realization in $5 \mathrm{G}$ networks," IEEE Communications Surveys \& Tutorials, vol. 23, no. 2, pp. 957-994, 2021.

[7] Y. Gao, "A survey study on the application of modern educational technology in English major college teaching in the age of 5G communication," Theory and Practice in Language Studies, vol. 11, no. 2, pp. 202-209, 2021.

[8] Y. Miao, L. Mao, and L. Gui, "Research on the integration of $5 \mathrm{G}+\mathrm{AI}$ technology to empower digital education under the background of industrial internet," CONVERTER, pp. 399407, 2021.

[9] Y. Noh and J. Y. Ro, "A study on the service provision direction of the National Library for Children and Young Adults in the 5G era," International Journal of Knowledge Content Development \& Technology, vol. 11, no. 2, pp. 77-105, 2021.

[10] L. Mishra and S. Varma, "Seamless health monitoring using 5G NR for Internet of Medical Things," Wireless Personal Communications, vol. 120, no. 3, pp. 2259-2289, 2021.

[11] S. Saafi, G. Fodor, J. Hosek, and S. Andreev, "Cellular connectivity and wearable technology enablers for industrial mid-end applications," IEEE Communications Magazine, vol. 59, no. 7, pp. $61-67,2021$. 
[12] H. N. Dai, Y. Wu, H. Wang, M. Imran, and N. Haider, "Blockchain-empowered edge intelligence for internet of medical things against COVID-19," IEEE Internet of Things Magazine, vol. 4, no. 2, pp. 34-39, 2021.

[13] L. Tian, "On the teaching reform of animation design major in higher vocational colleges," Journal of Frontiers in Art Research, vol. 1, no. 5, pp. 45-48, 2021.

[14] L. I. U. L. L. U. O. Jin-ling, "Research on the optimal allocation of the resources of the characteristic specialty group in higher vocational colleges," Computer \& Telecommunication, vol. 1, no. 3, pp. 6-8, 2020.

[15] P. Chen, "Innovation and exploration of course practice of animation major under the background of "Internet+"-a case study of "3D Animation Maya" course," World Scientific Research Journal, vol. 7, no. 5, pp. 500-508, 2021.

[16] D. Casella, F. Lo Torto, M. Marcasciano et al., "Breast animation deformity," Annals of Plastic Surgery, vol. 86, no. 5, pp. 512-516, 2021.

[17] J. H. Yan, B. C. Lee, and T. Yun, "A study on the elements of Chinese animation IP (intellectual property) development based on the pan-entertainment industry," International Journal of Internet, Broadcasting and Communication, vol. 13, no. 1, pp. 168-179, 2021.

[18] M. Takimoto, "A comparative study of animation versus static effects in the spatial concept-based metaphor awarenessraising approach on EFL learners' cognitive processing of request strategies," Language and Cognition, vol. 13, no. 2, pp. 191-226, 2021.

[19] J. Yee, "Puppets, gods, and brands: theorizing the age of animation from Taiwan, Teri Silvio (2019)," East Asian Journal of Popular Culture, vol. 7, no. 1, pp. 145-147, 2021.

[20] Z. T. Chen, "Poetic prosumption of animation, comic, game and novel in a post-socialist China: a case of a popular video-sharing social media Bilibili as heterotopia," Journal of Consumer Culture, vol. 21, no. 2, pp. 257-277, 2021. 